used the average of $\Sigma$ and $\Lambda$ masses since these are degenerate at the spherical level. ${ }^{7}$ )

We have derived here a large number of quadratic mass relations which agree with experiment to about $10 \%$ or better with essentially no free parameters. Earlier symmetry-breaking models, often quite accurate in restricted domains, cannot discriminate between quadratic and linear mass relations and, in fact, employ both.

Our results ${ }^{8}$ lend new support to the DTU approach and to its ability to generate predictions in both the meson and baryon sectors through a well-defined sequence of approximations.
${ }^{1}$ G. F. Chew and V. Poénaru, Lawrence Berkeley Laboratory Report No. LBL-9768, 1979 (to be published).

${ }^{2}$ G. F. Chew and C. Rosenzweig, Phys. Rep. 41C, 263 (1978).

${ }^{3}$ See, e.g., C. Schmid, D. M. Webber, and C. Sorensen, Nucl. Phys. B111, 317 (1976).

${ }^{4}$ We thank Dr. J. Finkelstein for comments on this point.

${ }^{5}$ H. M. Chan, J. Paton, and S. T. Tsou, Nucl. Phys. B86, 479 (1975).

${ }^{6} \mathrm{M}$ 。 Ademollo, G. Veneziano, and S. Weinberg, Phys. Rev. Lett. 22, 83 (1969).

${ }^{7}$ We thank L. A.P.Balazs for pointing this out to us.

${ }^{8}$ See also L. A. P. Balazs and B. Nicolescu, to be published.

\title{
Experimental Study of Heavy Charged Leptons and Search for Scalar Partners of Muons at PETRA $\left(12 \mathrm{GeV} \leqslant E_{\mathrm{c} . \mathrm{m} .} \leqslant 36.7 \mathrm{GeV}\right)$
}

D. P. Barber, U. Becker, G. D. Bei, G. Berghoff, A. Boehm, J. G. Branson, J. Bron, D. Buikman, J. D. Burger, M. Capell, C. C. Chang, H. S. Chen, M. Chen, C. P. Cheng, Y. S. Chu, R. Clare, P. Duinker, G. Y. Fang, Z. Y. Feng, H. S. Fesefeldt, D. Fong, M. Fukushima, J. C. Guo, A. Hariri, G. Herten, M. C. Ho, T. T. Hsu, D. Z. Jiang, R. W. Kadel, W. Krenz, J. Li, Q. Z. Li, D. Luckey, E. J. Luit, C. M. Ma, D. A. Ma, G. G. G. Massaro, T. Matsuda, H. Newman, M. Pohl, F. P. Poschmann, J. P. Revol, M. Rohde, H. Rykaczewski, I. Schulz, K. Sinram, M. Steuer, H. W. Tang, L. G. Tang, D. Teuchert, Samuel C. C. Ting, K. L. Tung, F. Vannucci, X. R. Wang, Y. X. Wang, J. Warnock, M. White, G. H. Wu, S. X. Wu, T. W. Wu, P. C. Yang, C. C. Yu, N. L. Zhang, and R. Y. Zhu

III Physikalisches Institut, Technische Hochschule, D-5100 Aachen, West Germany, and Deutsches ElektronenSynchrotron, D-2000 Hamburg 52, West Germany, and Laboratory for Nuclear Science, Massachusetts Institute of Technology, Cambridge, Massachusetts 02139, and Nationaal Instituut voor Kernfysica en Hogh-Energiefysica, 1009-DB Amsterdam, The Netherlands, and Institute of High Energy Physics, Chinese Academy of Science, Peking, People's Republic of China

(Received 2 October 1980)

Data from the MARK-J detector on the reactions $e^{+} e^{-} \rightarrow \mu^{+} \mu^{-}, \tau^{+} \tau^{-}$in the center-ofmass energy range from 12 to $36.7 \mathrm{GeV}$ are presented. The $\mu, \tau$ radii are shown to be $<10^{-16} \mathrm{~cm}$. A search has been made for the production of a new heavy lepton and for the production of spin-0 supersymmetric partners of the muon. 95\%-confidence-level lower limits of $16 \mathrm{GeV}$ for the mass of a new charged heavy lepton and $15 \mathrm{GeV}$ for the mass of the scalar partners of the muon are obtained.

PACS numbers: 14.60.-z, 13.10.+q

Since our previous publications ${ }^{1}$ in which we reported on the results of measurements of the reactions $e^{+} e^{-} \rightarrow \mu^{+} \mu^{-}$and $\tau^{+} \tau^{-}$in the center-ofmass energy range from 12 to $36.7 \mathrm{GeV}$, further data have been collected. We report here on our latest results on the verification of the validity of quantum electrodynamics (QED) with $\mu, \tau$ leptons, on the search for more heavy leptons, and on the search for spin-0 supersymmetric partners of muons.
The MARK-J detector and the method of detection of muons and $\tau$ leptons have been described previously. ${ }^{2}$ The data have been corrected for radiative effects ${ }^{3}$ and detector acceptance. The resultant $e^{+} e^{-}-\mu^{+} \mu^{-}$and $\tau^{+} \tau^{-}$cross sections, as a function of the c.m. energy $\sqrt{s}$, are plotted in Fig. 1 together with the QED predictions. The deviation of the measured cross section $\sigma$ from the QED prediction $\sigma_{\mathrm{QED}}$ is described with a form factor $F$ such that $\sigma=\sigma_{\mathrm{QED}} F^{2}(s)$, where $F(s)=1 \mp s /$ 


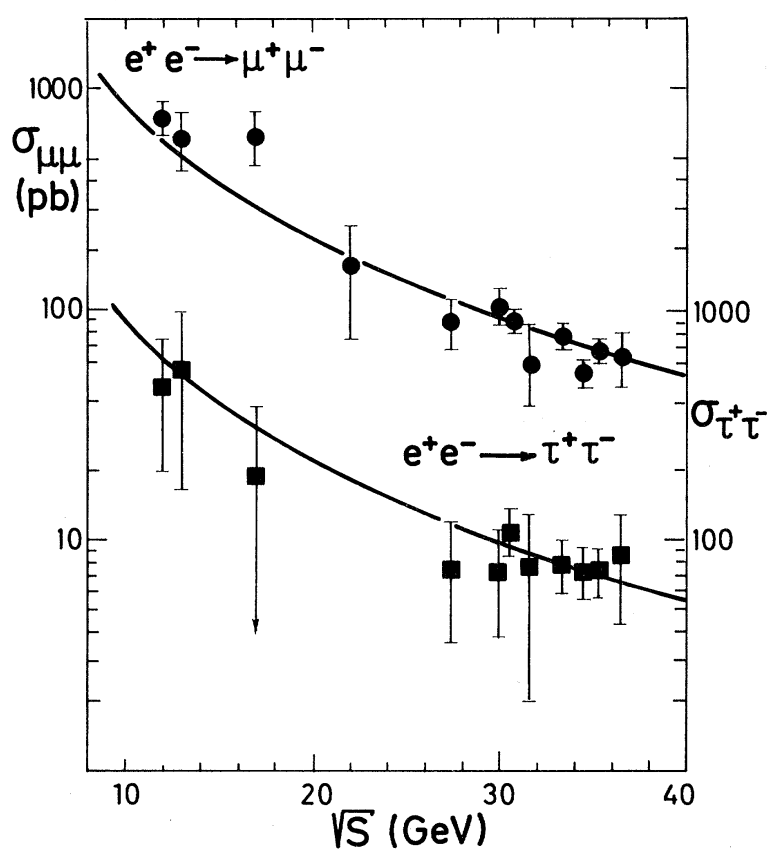

FIG. 1. Measured cross section for $e^{+} e^{-} \rightarrow \mu^{+} \mu^{-}$(left scale) and $e^{+} e^{-} \rightarrow \tau^{+} \tau^{-}$(right scale) corrected for radiative effects and acceptance, compared with the pointlike QED cross section.

$\left(s-\Lambda_{ \pm}{ }^{2}\right)$. The leptonic form factor $F(s)$ enables us to parametrize the maximum permissible radii of the leptons. Note that the neutral weak current (via $Z^{0}$ ), which is mainly axial, does not contribute to the first-order total cross section. The resulting 95\%-confidence-level lower limits on the cutoff parameters, $\Lambda$, are listed in Table I. From the uncertainty principle, the cutoff parameters at highest PETRA energies imply that the muon and $\tau$ lepton are pointlike particles with radii smaller than $10^{-16} \mathrm{~cm} .^{1,4}$

We have also searched for new heavy leptons. Following the initial searches ${ }^{5}$ for the $\tau$ lepton, the discovery ${ }^{6}$ and the further study of its properties, ${ }^{7}$ there has been great interest in searching for a new heavy lepton, which would extend the series $e, \mu, \tau$. Analogous to the $\tau$ lepton we assume that a new heavy lepton (HL) couples universally to leptons and quarks according to the standard $V-A$ weak-interaction theory and has the following decay modes: $\tau^{-} \bar{\nu}_{\tau} \nu_{\mathrm{HL}}(9.2 \%), \mu-\bar{\nu}_{\mu}$ $+\nu_{\mathrm{HL}}(10.6 \%), e^{-} \bar{\nu}_{e} \nu_{\mathrm{HL}}(10.6 \%)$, and hadrons $+\nu_{\mathrm{HL}}(69.6 \%)$. The branching ratios, ${ }^{8}$ which are mass dependent, are given here for a heavy-lepton mass of $14 \mathrm{GeV}$. Because of their large mass and low velocity, the decay products would be ex-
TABLE I. Cutoff parameters in gigaelectronvolts for muons and $\tau$ leptons.

\begin{tabular}{ccc}
\hline \hline Lepton & $\Lambda_{-}(\mathrm{GeV})$ & $\Lambda_{+}(\mathrm{GeV})$ \\
\hline$\mu$ & 129 & 192 \\
$\tau$ & 127 & 100 \\
\hline
\end{tabular}

pected to have large angles with respect to the HL line of flight. This contrasts to the decay products of the $\tau$ at PETRA energies which are tightly collimated. Heavy-lepton production is most easily recognized in the MARK-J detector for events in which one lepton decays into a muon and neutrinos and the other lepton decays into hadrons and a neutrino. Hadrons are detected by their energy deposit, $E_{\text {vis, }}$, in the calorimeter.

Heavy-lepton candidates with masses greater than $6 \mathrm{GeV}$ are selected by applying the following criteria: (1) $0.2 E_{\text {Beam }}<E_{\text {vis }}<E_{\text {Beam, }}$ (2) acoplanarity $>30^{\circ}$, (3) $E_{K}>0.1 E_{\text {vis }}$, (4) charged multiplicity of events $>2$, and (5) $30^{\circ}<\theta_{H}<150^{\circ}$. The "acoplanarity" is defined as the absolute value of $\left|180^{\circ}-\delta \varphi\right|$, where $\delta \varphi$ is the angle between the muon momentum vector and the total energy flow vector $^{2}$ of the hadrons $\overrightarrow{\mathrm{E}}_{H}$ projected on a plane perpendicular to the beam line. $E_{K}$ is the energy deposited in the outer part of the calorimeter and $\theta_{H}$ is the polar angle between $\overrightarrow{\mathrm{E}}_{H}$ and the beam line. Cut 1 reduces the background from $e^{+} e^{-}$ $\rightarrow$ hadrons; Cut 2 suppresses the contribution from $e^{+} e^{-} \rightarrow \tau^{+} \tau^{-}$; and Cut 3 helps to select hadronic showers as opposed to electromagnetic showers. Cuts $1-5$ are also effective in eliminating background from the "two-photon" process, ${ }^{9}$ $e^{+} e^{-} \rightarrow e^{+} e^{-} \mu^{+} \mu^{-}$, and from $e^{+} e^{-} \rightarrow \mu^{+} \mu^{-} \gamma$ where a high-energy photon is radiated at large angles.

No event was observed in the data with $33 \leqslant \sqrt{s}$ $\leqslant 36.7 \mathrm{GeV}$, corresponding to a time-integrated luminosity of $6.9 \mathrm{pb}^{-1}$. The number of events predicted by a Monte Carlo calculation as a function of the heavy-lepton mass $M_{\mathrm{HL}}$ is shown in Fig. 2 along with the $95 \%$-confidence-level upper limit from the data. Figure 2 demonstrates that the existence of a sequential heavy lepton with a mass between 6 and $16 \mathrm{GeV}$ is excluded.

Heavy leptons with $M_{\mathrm{HL}}<6 \mathrm{GeV}$ would decay into final states similar in appearance to those from $\tau$ decay, and would tend to be included in our sample of $e^{+} e^{-} \rightarrow \tau^{+} \tau^{-}$events. The inset in Fig. 2 shows the Monte Carlo prediction for the total number of events in the $\tau$ sample from $e^{+} e^{-}$ $\rightarrow \tau^{+} \tau^{-}$(52) and from heavy-lepton production, as 


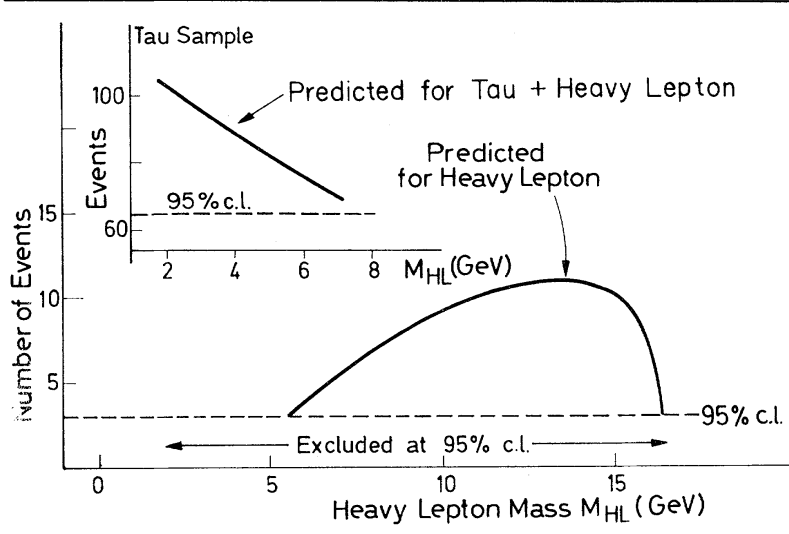

FIG. 2. Number of events expected for the production of a new (sequential) heavy lepton as a function of mass. The inset shows the number of events expected in the $\tau$ sample from $\tau$ - and heavy-lepton production. We observe a total of $54 \tau$ events. The dashed line corresponds to the $95 \%$-confidence-level upper limits for $\tau$ events.

a function of $M_{\mathrm{HL}}$. The inset demonstrates that with more than $95 \%$ confidence we exclude the existence of the heavy lepton with mass $M_{\mathrm{HL}}<6$ $\mathrm{GeV}$. We are thus able to rule out the existence of a new heavy lepton for a $M_{\mathrm{HL}}<16 \mathrm{GeV}$.

In the framework of supersymmetric theories, ${ }^{10}$ spin-0 partners of the muon are expected to decay only according to the reactions

$$
\begin{aligned}
& s_{\mu^{-}} \rightarrow \mu^{-}+\text {photino (goldstino) } \\
& t_{\mu}{ }^{-} \rightarrow \mu^{-}+\text {antiphotino (antigoldstino), }
\end{aligned}
$$

where $s_{\mu}$ and $t_{\mu}$ are the spin-0 partners of the muon associated with the left- and right-handed parts of the muon field, respectively, the photino is the spin- $\frac{1}{2}$ partner of the photon and the goldstino is the spin- $\frac{1}{2}$ particle associated with the spontaneous breaking of supersymmetry. Since $s_{\mu}$ and $t_{\mu i}$ carry unit electric charge they may be produced in pairs in $e^{+} e^{-}$annihilation according to the cross section

$$
\begin{aligned}
\frac{d \sigma\left(e^{+} e^{-} \rightarrow s_{\mu}{ }^{-} s_{\mu}{ }^{+} \text {or } t_{\mu}{ }^{-} t_{\mu}{ }^{+}\right)}{d(\cos \theta)} & =\frac{\pi \alpha^{2} \beta^{3} \sin ^{2} \theta}{4 s}, \\
\beta & =\left[1-(m / E)^{2}\right]^{1 / 2},
\end{aligned}
$$

which is characteristic of spin-0 particle production. $m$ is the mass of $t_{\mu}$ or $s_{\mu}, E$ is the beam energy, and $\theta$ is the scattering angle.

Because of the uniqueness of the decay reactions (1a) and (1b), the extremely short lifetime ${ }^{10}$ of $s_{\mu}$ and $t_{\mu}$ and the prediction that the interaction cross section of photino and goldstino are

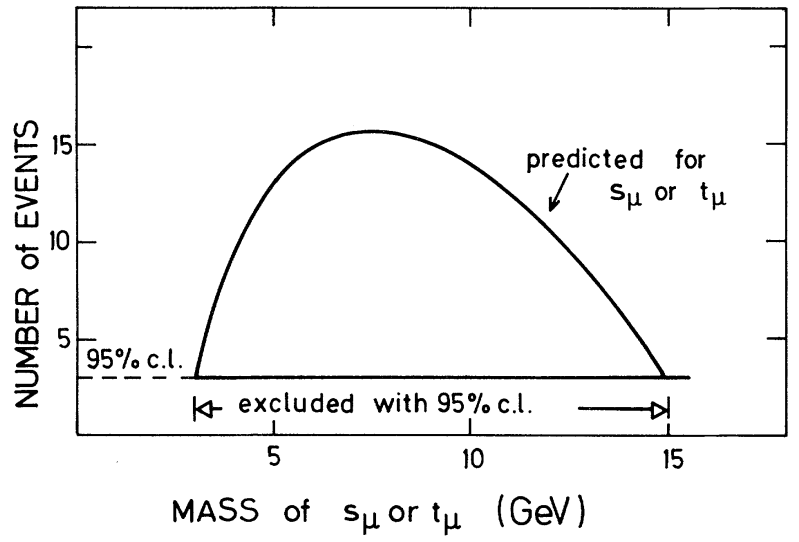

FIG. 3. Number of events expected for the production of a spin-0 partner $s_{\mu}$ or $t_{\mu}$ of the muon as a function of mass. The upper limit of events (at $95 \%$ confidence level) and the mass range excluded is also indicated.

expected to be very small, ${ }^{11}$ only muon pairs are observed in the final state. Near threshold production of $s_{\mu}$ and $t_{\mu}$ the two residual muons would be produced isotropically in space. Data from SPEAR place a lower limit ${ }^{12}$ of $3.5 \mathrm{GeV}$ on the mass of $s_{\mu}$ and $t_{\mu}$. Thus, over the PETRA energy range of 12 to $36.7 \mathrm{GeV}$, an increase in the production of acoplanar muon pairs should be observed if a new threshold is passed.

A set of criteria for selection of such muon pairs has been established which eliminate events from the processes

$$
\begin{aligned}
& e^{+} e^{-} \rightarrow \mu^{+} \mu^{-}, \quad \mu^{+} \mu^{-} \gamma, \\
& e^{+} e^{-} \rightarrow \tau^{+} \tau^{-}, \tau^{+} \tau^{-} \gamma, \\
& e^{+} e^{-} \rightarrow e^{+} e^{-} \mu^{+} \mu^{-},
\end{aligned}
$$

which are the main sources of background. 8,9 To reject this background we select events with the following criteria: (1) acoplanarity $\geqslant 20^{\circ}$, (2) electromagnetic energy $\leqslant 20 \% E_{\text {beam }}$, and (3) muon momenta $\geqslant 20 \% E_{\text {beam }}$. No event was observed in the energy range $33 \mathrm{GeV} \leqslant \sqrt{s} \leqslant 36.7 \mathrm{GeV}$.

Figure 3 shows the expected number of events predicted by a Monte Carlo calculation for the production of $s_{\mu}$ or $t_{\mu}$ pairs. We also indicate the number of events corresponding to the $95 \%$ confidence level.

Thus, the data show that no $s_{\mu}$ - or $t_{\mu}$-type particle is produced with a mass between 3 and 15 $\mathrm{GeV}$. If $s_{\mu}$ and $t_{\mu}$ particles have the same mass and are both produced, the number of expected events is twice as large. In this case they can both be excluded with more than $99 \%$ confidence. 
In conclusion, our results are as follows:

(1) The muon and $\tau$ leptons are pointlike particles with a charge radius smaller than $\sim 10^{-16} \mathrm{~cm}$ (at $95 \%$ confidence level).

(2) The existence of a new charged sequential heavy lepton is excluded with $95 \%$ confidence level for a mass lower than $16 \mathrm{GeV}$.

(3) The production of a scalar supersymmetric partner of the muon is excluded with $95 \%$ confidence level for a mass lower than $15 \mathrm{GeV} \cdot{ }^{13}$

We wish to thank Professor H. Schopper, Professor E. Lohrmann, Professor G. Voss, Dr. F. J. Eppling, and Dr. G. Soehngen, for their valuable support.

We acknowledge helpful discussions with Professor P. Fayet, Professor J. J. Sakurai, Professor D. Schildknecht, and Professor P. Zerwas. We also thank P. Berges, S. Burger, S. Marks, and $\mathrm{D}$. Osborne for technical and administrative help.

${ }^{1}$ D. P. Barber et al ., Phys. Rev. Lett. 42, 1110 (1979), and 43, 1915 (1979), and Phys. Lett. 95B, 149 (1980).

${ }^{2}$ The MARK-J Collaboration, Phys. Rep. 63, 337-391 (1980).

${ }^{3}$ F. A. Berends, K. J. F. Gaemers, and R. Gastmans, Nucl. Phys. B57, 381 (1973), and B63, 381 (1973), and B68, 541 (1974); F. A. Berends and G. J. Komen, Phys. Lett. 63B, 432 (1976). We used Monte Carlo simulations of $e^{+} e^{-} \rightarrow \mu^{+} \mu^{-} \gamma$ and $e^{+} e^{-} \rightarrow \tau^{+} \tau^{-} \gamma$, correct to order $\alpha^{3}$, based on the recent work described by F. A. Berends and R. Kleiss, DESY Report No. 80/66 (unpublished).

${ }^{4}$ A. Boehm, Technische Hochschule, Aachen, Report No. PITHA 80/9, 1980 (unpublished); W. Bartel et al.,
Phys。Lett. 92B, 206 (1980); Ch. Berger et al ., Z. Phys. C 4, 269 (1980), and Phys. Lett. 94B, 87 (1980); R. Brandelik et al ., Phys. Lett. 94B, 259 (1980), and 92B, 199 (1980).

${ }^{5} \mathrm{ADONE}$, Istituto Nazionale di Fisica Nucleare Report No. INFN/AE-67/3, 1967 (unpublished); M. Bernardini et al ., Nuovo Cimento 17A, 383 (1973); S. Orito et al., Phys. Lett. 48B, 165 (1974).

${ }^{6}$ M. L. Perl, Phys. Rev. Lett. 35, 1489-1492 (1975); G. Feldman et al., Phys. Rev. Lett。38, 117 (1977).

${ }^{7}$ For a review of our present knowledge of the $\tau$ lepton, see G. Fluegge, Z. Phys. C 1, 121 (1979), and the references therein. R. Brandelik etal., Phys. Lett. 92B, 199 (1980).

${ }^{8}$ Y.-S. Tsai, SLAC Report No. SLAC-PUB-2450, 1979 (unpublished). Note that the $\tau$ branching ratios are taken from the compilations of world data in Ref. 7. The $\tau \rightarrow \mu \nu \nu$ branching ratio is taken as $17.0 \%$.

${ }^{9}$ All Monte Carlo calculations concerning $e^{+} e^{-}$ $\rightarrow e^{+} e^{-} \mu^{+} \mu^{-}$were made using J. A. M. Vermaseren's event generator, and a detailed simulation of the MARK$\mathrm{J}$ detector.

${ }^{10} \mathrm{Yu}$. A. Gol'fand and E. P. Likhtman, Pis'ma Zh. Eksp. Teor. Fiz. 13, 452 (1971) [JETP Lett. 13, 323 (1971)]. J. Wess and B. Zumino, Nucl. Phys. B70, 39 (1974); P. Fayet and S. Ferrara, Phys. Rep. 32C, 249 (1977); P. Fayet, Phys. Lett. 69B, 489 (1977); G. R. Farrar and P. Fayet, Phys. Lett. 76B, 575 (1978), and 79B, 442 (1978); P. Fayet, Phys. Lett. 84B, 421 (1979); G. R. Farrar and P. Fayet, Phys. Lett. 89B, 191 (1980).

${ }^{11}$ P. Fayet, Phys. Lett. 86B, 272 (1979).

${ }^{12}$ F. B. Heiel et al ., Nucl. Phys. B138, 189 (1978). ${ }^{13} \mathrm{~A}$ search for the scalar supersymmetric partners of the electron is presented by H. Spitzer, DESY Report No. 80/43, 1980 (unpublished). For a discussion of preliminary work on searches for a heavy lepton, see D. Cords, in Proceedings of the Twentieth International Conference on High Energy Physics, Madison, Wisconsin, July, 1980 (to be published). 